\section{P94 (continued)}

Measurable Outcome/Analysis: Agreement statements were measured to determine the impact on knowledge and use of the videos. Additional questions captured participant feedback on the video training modules. Survey results were analyzed using SPSS (version 25) to assess differences between groups. A Mann Whitney Test was used to compare the survey responses between groups.

Results: There was a significant increase $(P<.05)$ between group $1(\mathrm{n}=34)$ and group $2(\mathrm{n}=34)$ on how strongly they agree that the videos increased their knowledge pertaining to causes of foodborne illness and human risk. There was a significant increase $(P<.05)$ between groups on how strongly they agree on ease of video use. There was a significant decrease $(P<.05)$ between groups on how strongly they agree that the pace of the videos was too fast.

Conclusions: Overall, participants agreed that the revised videos provided more useful information and were easier to use compared to the draft videos.

Funding: USDA, California Department of Food and Agriculture and California State University Agricultural Research Initiative.

\section{Basic Food and Nutrition Knowledge}

\section{P95 Reliability of the Food Literacy Assessment Tool (FLAT) in Low-Income Adults}

Audrey Hemmer, BS, University of Cincinnati; Carmen Fightmaster, BS, RN, University of Cincinnati; Youn Seon Lim, PhD, University of Cincinnati; Melinda Butsch Kovacic, PhD, MPH, University of Cincinnati; Seung-Yeon Lee, PhD, lee2so@uc.edu, University of Cincinnati, 3225 Eden Ave, Cincinnati, $\mathrm{OH}, 45267$

Background: Food literacy is defined as the knowledge, skills, and behaviors required to plan and manage, select, prepare, and eat in support of health within cultural, socioeconomic, and environmental context. Low-income populations show disproportionately higher prevalence of diet-related chronic diseases compared to their counterparts. Nutrition plays a key role in prevention of these chronic diseases. There is no valid and reliable tool to assess food literacy in low-income populations. The Food Literacy Assessment Tool was developed to assess knowledge, self-efficacy, and behavior related to food literacy, and was previously tested for face validity and content validity.

Objective: To evaluate the reliability of the Food Literacy Assessment Tool (FLAT).

Study Design, Setting, Participants: A convenience sample of low-income adults were recruited at a nonprofit organization providing emergency aid through a food pantry, charitable pharmacy, and social services to clients in need. Ninety-eight adults completed the FLAT, and 75 of the 98 adults returned and completed the same survey a second time $1-3$ weeks after their initial survey.
Measurable Outcome/Analysis: Internal consistency reliability was evaluated using Kuder-Richardson Formula 20 (KR-20) for knowledge (dichotomous variable) and Cronbach's $\alpha$ for self-efficacy and behavior (measured using Likert scales). Test-retest reliability was assessed using intraclass correlation coefficient (ICC).

Results: The FLAT was found to have acceptable internal consistency in self-efficacy (Cronbach's $\alpha=.921$ ) and behavior (Cronbach's $\alpha=.899$ ), but not in knowledge (KR$20=.510)$. The FLAT also showed acceptable test-retest reliability for knowledge $(\mathrm{ICC}=.839)$, self-efficacy $(\mathrm{ICC}=.703)$, and behavior $(\mathrm{ICC}=.930)$.

Conclusions: Overall, the FLAT is considered to be appropriate to assess food literacy in low-income adults in the United States.

Funding: NIH.

\section{P96 Your Child is Overweight, Now What? Understanding How North Carolina Head Start Staff Communicate with Families about Childhood Obesity}

Dana Shefet, shefetd17@students.ecu.edu, East Carolina University, 2150 West 5th St, Suite 2307, Greenville, NC, 27858; Beth Miller, PhD, LD, RDN, Miami University; Stephanie Nicely, EdD, MSN, RN, Miami University; Kara Trimbach, Miami University; Virginia Stage, PhD, RDN, East Carolina University

Background: Children (3-5 years) enrolled in Head Start (HS) are at increased risk for obesity. In an effort to prevent childhood obesity, HS implemented a Body Mass Index screening program in 2012. This program provides opportunities for HS to engage families by communicating information about children's weight status; however, limited research is available to describe the barriers and facilitators HS staff face when communicating this information to families.

Objective: Explore common experiences of HS Health/ Nutrition Managers when communicating information to families about their child's weight status.

Study Design, Setting, Participants: Researchers conducted 15 in-depth telephone interviews with Managers across North Carolina.

Measurable Outcome/Analysis: Phenomenology was used to guide study design and analysis. Interviews were recorded and transcribed verbatim. Researchers identified significant statements through open-coding and grouped them into themes focusing "what" and "how" participants experienced the phenomenon.

Results: Researchers identified 3 themes: Process of Communication; Barriers to Communication; and Communication Supports. Programs used different methods to communicate about children's weight (eg, letters, meetings, no communication). Parent reactions to children's weight status often dictated communication methods chosen; negative reactions often resulted in limited communication. Managers perceived parents' backgrounds (socioeconomic status, education) and the "shame" asso- 